\title{
UV optical measurements of the Nozomi spacecraft interpreted with a two-component LIC-flow model (Corrigendum)
}

H. Nakagawa ${ }^{1}$, M. Bzowski ${ }^{2}$, A. Yamazaki ${ }^{3}$, H. Fukunishi ${ }^{4}$, S. Watanabe ${ }^{5}$, Y. Takahashi ${ }^{1}$, M. Taguchi ${ }^{6}$, I. Yoshikawa $^{7}$, K. Shiomi ${ }^{8}$, and M. Nakamura ${ }^{3}$

1 Department of Geophysics, University of Tohoku, 980-8577 Miyagi, Sendai, Japan e-mail: rom@pat.gp.tohoku.ac.jp

2 Space Research Centre PAS, Bartycka 18A, 00-716 Warsaw, Poland e-mail: bzowski@cbk.waw.pl

${ }^{3}$ Institute of Space and Astronautical Science, Japan Aerospace Exploration Agency, 229-8510 Kanagawa, Sagamihara, Japan

4 Japan Society for the Promotion of Science, 100084 Beijing, PR China

5 Department of Earth and Planetary Science, University of Hokkaido, 001-0021 Hokkaido, Japan

${ }^{6}$ Department of Physics, College of Science, Rikkyo University, 171-8501 Tokyo, Japan

7 Department of Earth and Planetary Science, University of Tokyo, 113-0033 Tokyo, Japan

8 Earth Observation Research Center, Japan Aerospace Exploration Agency, 305-8505 Ibaraki, Tsukuba, Japan

A\&A 491, 29-41 (2008), DOI: 10.1051/0004-6361:20079241

Key words. Sun: UV radiation - ISM: atoms - ultraviolet: ISM - errata, addenda

We found a typographical error regarding the uncertainty of the helium flow direction in our published paper Nakagawa et al. (2008). The helium flow direction should be $\left(258.7 \pm 8.0^{\circ}, 3.4 \pm 8.0^{\circ}\right)$ instead of $\left(258.7 \pm 3.4^{\circ}\right)$. As a consequence, the description for the helium flow direction in page 39 has to be replaced by "Residuals of simulated intensities with respect to observations attained minimum values for a downwind direction of $\left(78.78 \pm 8.0^{\circ},-3.48 \pm 8.0^{\circ}\right)$, corresponding to an upwind direction of $(258.78 \pm$ $\left.8.0^{\circ}, 3.48 \pm 8.0^{\circ}\right)$." All conclusions of the original paper remain unaffected. H. Nakagawa is grateful to Priscilla Frisch for pointing out this error.

\section{References}

Nakagawa, H., Bzowski, M., Yamazaki, A., et al. 2008, A\&A, 491, 29 